\title{
Prediction of potential spread areas of African swine fever virus through wild boars using Maxent model
}

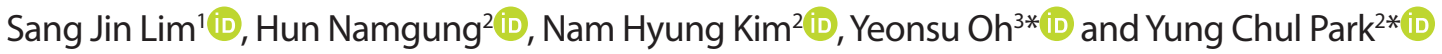 \\ IInstitute of Forest Science, Kangwon National University, Chuncheon 24341, Republic of Korea \\ 2Division of Forest Science, College of Forest \& Environmental Sciences, Kangwon National University, Chuncheon 24341, Republic of Korea \\ ${ }^{3}$ College of Veterinary Medicine \& Institute of Veterinary Science, Kangwon National University, Chuncheon 24341, Republic of Korea
}

\author{
ARTICLE INFO \\ Received January 11, 2022 \\ Revised February 10, 2022 \\ Accepted February 10, 2022 \\ Published on March 3, 2022 \\ *Corresponding author \\ Yung Chul Park \\ E-mail parky@kangwon.ac.kr \\ Yeonsu Oh \\ E-mail yeonoh@kangwon.ac.kr
}

\begin{abstract}
Background: In South Korea, African swine fever virus (ASFV) has spread among wild boars through Gangwon-do to Dangyang-gun, Chungcheongbuk-do on the southern border of Gangwon-do. To prevent the spread of ASFV to African swine fever (ASF)-free areas, it is necessary to identify areas with a high probability of finding ASFV-infected carcasses and to reduce the density of wild boars in those areas. In this study, we described the propagation trend of ASFV among wild boars, constructed the habitat suitability maps for ASFV-infected carcasses, and suggested areas with a high probability of finding ASFV-infected carcasses and an important route of ASFV transmission.

Results: Despite the active quarantine policies in Korea to prevent the spread of ASFV through wild boars, there was no significant difference in the monthly average of number of ASFV-infected carcasses observed between 2020 and 2021. The ASFV-infected carcasses were found more in winter and spring (January to April). Since the first ASF outbreak in wild boars on October 2, 2019, the maximum width of ASFV-infected carcass distribution area was $222.7 \mathrm{~km}$ for about 26 months till November 20, 2021. The habitat suitability map, based on GPS coordinates of ASFV-infected wild boar carcasses, shows that highly detectable areas of ASFV-infected carcasses were sporadically dispersed in western and southwestern parts of Gangwon-do, and ranged from north to south of the province along the Baekdudaegan Mountains, whereas poorly detectable areas ranged along the north to the south in the middle parts of the province.

Conclusions: Our suitability model, based on the GPS coordinates of ASFV-infected carcasses, identifies potential habitats where ASFV-infected carcasses are likely to be found and ponential routes where ASFV is likely to spread. Among ASF-free areas, the areas with high suitability predicted in this study should be given priority as survey areas to find ASFV-infected carcasses and hunting areas to reduce wild boar populations.
\end{abstract}

Keywords: African swine fever virus, wild boar carcass, Sus scrofa, habitat suitability index, ASF spread pattern

\section{Introduction}

African swine fever (ASF) is an emerging contagious viral disease which affects both domestic pigs and wild boars (EFSA et al. 2021). The disease is caused by African swine fever virus (ASFV) which is the sole member of the family Asfarviridae and is an enveloped double-stranded DNA virus (Dixon et al. 2013; Mazur-Panasiuk et al. 2019). Due to high stability to environmental change, ASFV can remain infectious for several months in variable environments (Fischer et al. 2020; Mazur-Panasiuk et al. 2019). ASFV can be transmitted via direct ASFV-infected animal contact (Mazur-Panasiuk et al. 2019; Olesen et al. 2017), infected carcasses (Chenais et al. 2019; Probst et al. 2019), fomites (Mazur-Panasiuk et al. 2019; Mur et al. 2012), and infected soft ticks of the genus Ornithodoros (Mazur-Panasiuk et al. 2019; Plowright et al. 1969). ASF has been endemic to sub-Saharan Africa since the 1920s. In Europe, ASF spread widely to parts of European countries after outbreaks in Georgia in 2007, which was then reported in 11 European members (Lithuania, Poland, Latvia, Estonia, Czechia, Romania, Hungary, Bulgaria, Slovakia, Belgium, and Greece) (EFSA et al. 2021), and in the European, middle and south-eastern parts of Russian Federation (Zakharova et al. 2021).

In Asia and the Pacific, since the 1st occurrence of ASF 
in China on August 1, 2018, the disease has spread to Mongolia (January, 2019), Vietnam (February, 2019), Cambodia (March, 2019), Hong Kong (May, 2019), North Korea (May, 2019), Laos (June, 2019), Myanmar (August, 2019), Philippines (July, 2019), South Korea (September, 2019), TimorLeste (September, 2019), Indonesia (November, 2019), Papua New Guinea (March, 2020), and more recently in India (May, 2020). Moreover, Russia reported an ASF event in the east side of the country (bordering China) in August 2019 (OIE 2021).

In South Korea, the first case of ASF was reported in a domestic pig farm in Paju-si which belongs to Gyeonggi-do on September 16, 2019, and then in wild boars, the disease spread through Gangwon-do to Dangyang-gun, Chungcheongbuk-do, on the southern border of Gangwon-do. Gangwon-do covers the largest part of the Baekdudaegan Mountains and the forest area of Gangwon-do occupies $80.5 \%$ of the province (Korea Forest Service 2021). The mountains in Gangwon-do are connected to those in Chungcheongbuk-do through the Baekdudaegan Mountains (Korea Forest Service 2021). In order to prevent the spread of African swine fever virus (ASFV) to ASF-free areas, it is necessary to identify areas with a high probability of finding ASFV-infected carcasses and reduce the density of wild boars in those areas. The habitat suitability map (HSM), which is derived using the GPS coordinates of the sites where ASFV-infected carcasses were found, provides information on the area to be surveyed for searching the ASFV-infected carcasses in ASF-free areas. It can provide important information in selecting the areas which should be managed in order to prevent the spread of ASFV to ASF-free areas. If ASFV spreads to the ASF-free areas, it is more likely that ASFV-infected carcasses will be found first in the areas with more suitable habitats in the HSM. Thus,
HSM can help significantly in selecting hunting target areas, ASFV-infected carcass search areas and fencing installation areas.

In this study, we have described the propagation trend of ASFV among wild boars, constructed habitat suitability maps for ASFV-infected carcasses in Gangwon-do using the GPS coordinates of the sites where ASFV-infected carcasses were found, and suggested the areas with a high probability of spreading ASFV to ASF-free areas.

\section{Materialls and Methods}

\section{Study area and GPS data collection}

Gangwon-do is located in the northeastern South Korea and consists of 7 cities and 11 counties. The province borders Gyeonggi-do to the west and Chungcheongbuk-do to the south. To analyze the trend of ASF spread in wild boars, 1710 GPS coordinates of ASFV-infected wild boar carcasses (Fig. 1A), which were detected during the period of October 2, 2019 to November 20, 2021, and the report dates were provided from Ministry of Agriculture, Food and Rural Affairs, Republic of Korea.

We constructed two types of HSM for wild boars in Gangwon-do: for ASFV-infected wild boars found dead (ASF-infected carcass) and for hunted wild boars in ASFfree areas. For the former map, we used the GPS coordinates of the 1,646 sites of the ASFV-infected carcasses found in Gangwon-do. For the latter map, we used the GPS coordinates of the 448 sites of hunted wild boars collected in hunting grounds in Hongcheon-gun, Hoengseong-gun, and Pyeongchang-gun which were located in the southern and western parts of Gangwon-do during the period from February 3, 2021 to March 30, 2021, before the ASF out-
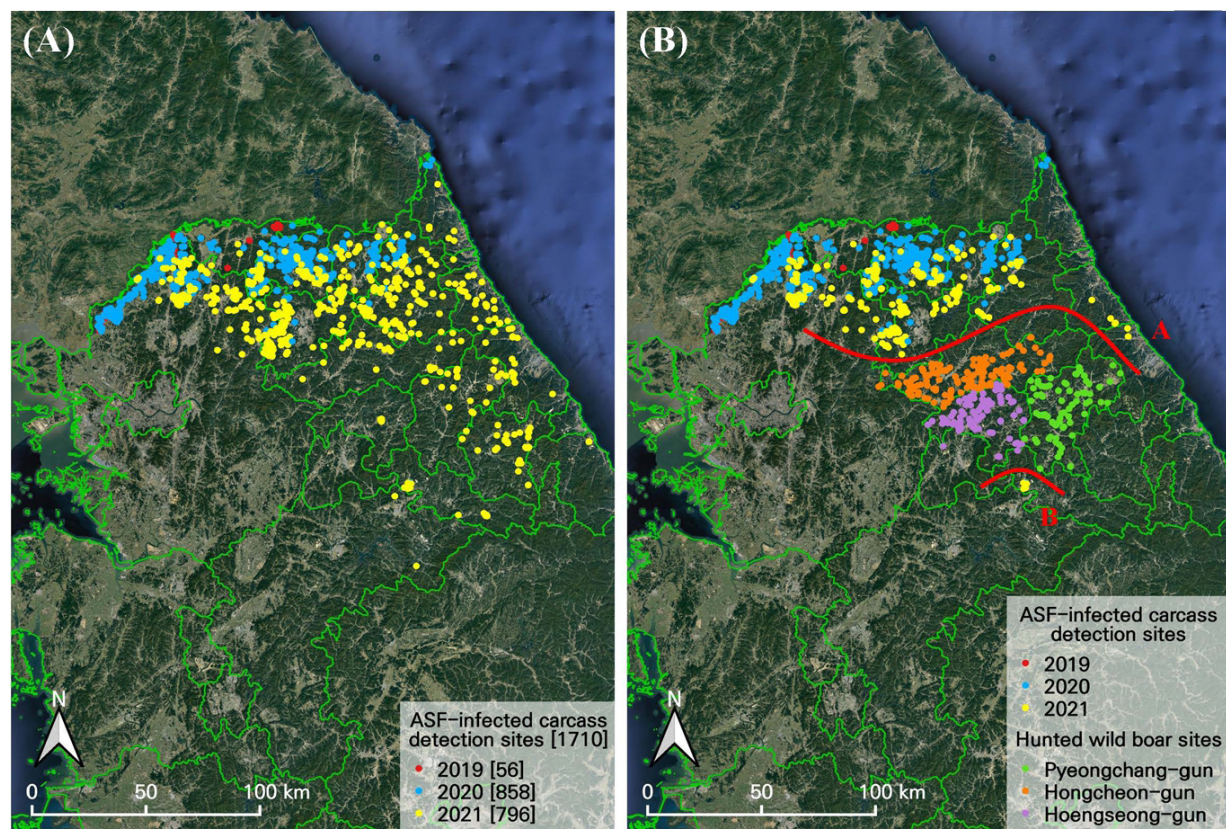

Fig. 1 Localities of sample collection. The 1,710 sites where African swine fever virus (ASFV)-infected wild boar carcasses were found from October 2, 2019 to November 20, 2021 (A) and the points above the line $A$ and those below the line B indicate the sites where ASFV-infected carcasses were found from October 2, 2019 to March 30, 2021 (B). The points between the lines A and B indicate the 448 sites where wild boars were hunted in the hunting area from February 3, 2021 to March 30, 2021 (B). ASFV-infected carcasses were not found in the hunting area until March 30, 2021. 
break in the hunting grounds (Fig. 1B).

\section{Habitat suitability modeling}

The HSMs were constructed through Maxent using occurrence records and environmental variables. The GPS coordinates of the sites where ASFV-infected carcasses were found and the sites where hunted wild boars were found were recorded in the CSV file format. The CSV file was used as the input file for Maxent. In addition to the species occurrence data, 11 variables in total were also used as model inputs, referring to the previous studies (Lim et al. 2015; Seo et al. 2008; Song and Kim 2012). The variables were categorized into terrain-, forest- and human-disturbed variables. The three terrain variables (elevation, aspect, and slope) were obtained from the Digital Elevation Model (DEM) database in the National Geographic Information Institute website (http://map.ngii.go.kr/) and one terrain variable (distance from water system) was obtained from the stream order map of the Water Resources Management Information System (WRMIS). The four forest variables (forest type, crown density, age-classes of trees, and diameter at breast height of trees) were taken from the 1:5,000 Forest Type Map by the Korea Forest Service. The two human-disturbed variables (distance from residences and distance from farmlands) were from the 2020 Subdivision Land Cover Map from Ministry of Environment and one human-disturbed variable (distance from roads) was from the Road Map of Korea from Ministry of Land.

To understand the spatial distribution throughout Gangwon-do, the ESRI shape-file of the administrative boundary map along the province was overlaid onto the grid file containing bioclimatic variables in ArcGIS 10.2 (Esri, Redlands, CA, USA). This grid file was then overlaid on DEM data to generate information on the altitudinal range of wild boars in the area.

\section{Resullts and Discussion}

\section{Spread pattern of ASFV-infected wild boar carcasses}

After the first outbreak in a domestic pig farm in Paju-si, Gyeonggi-do on September 16, 2019, an ASFV-infected wild boar carcass was first discovered in Sinseo-myeon (GPS coordinate; 38.264194, 127.077194), Yeoncheon-gun, Gyeonggi-do on October 2, 2019. In Gangwon-do, an ASFV-infected wild boar carcass was first discovered in Wonnam-myeon $(38.309722,127.603056)$, Cheorwon-gun, which borders Gyeonggi-do, on October 11, 2019, and then found across wide regions of the northern Gangwon-do from the western (Cheolwon-gun) to eastern (Goseonggun) regions. In 2020, ASFV-infected carcasses were found only in the northern Gangwon-do, and not in the middle regions of the province. Unexpectedly, however, on De- cember 28, 2020, an ASFV-infected carcass was found in Jucheon-myeon, Yeongwol-gun in the southernmost part of Gangwon-do, far away from previously known outbreak areas.

In 2021, ASF-infected carcasses were found more widely in a region from the northern part to the southeastern part of Gangwon-do. In the Yeongseo region, which is located in the western part of the Baekdudaegan Mountains in Gangwon-do, up to the northern outskirts of Chuncheon, ASFV-infected carcasses were found frequently (176 cases). The ASFV-infected carcasses (31 cases) were also found in Hongcheon and Hoengseong (Fig. 1A).

In Yeongwol-gun (Satgat-myeon), which is located in the southernmost part of Gangwon-do, adjacent to Chungcheongbuk-do, an ASFV-infected carcass was discovered again on October 31, 2021, almost 10 months after the first incidence in Yeongwol-gun (Jucheon-myeon) on December 28,2020 . Since then, 8 more cases were found in Yeongwol-gun in less than a month until November 20, 2021.

In Chungcheongbuk-do, the first case of ASFV-infected carcasses was reported in Danyang-gun, which is adjacent to Yeongwol-gun, Gangwon-do, on November 14, 2021; later they were also found in Jecheon-si on November 19, 2021, showing a trend of spreading in Chungcheongbukdo.

Despite the active quarantine policies in Korea to block the spread of ASFV through wild boars, such as fencing and pre-emptive hunting, and detection and removal of ASFV-infected carcasses, ASF is currently spreading at a relatively rapid pace among wild boars. The maximum propagation linear distance of ASF in wild boars was 222.7 km (from Paju-si; 37.912916, 126.711692 to Samcheok-si; $37.427001,129.160967)$ for about 26 months up to November 20, 2021 since the first ASF outbreak in wild boars on October 2, 2019. Since ASF spread by wild boar movement was estimated to be $<25 \mathrm{~km}$ in a year (Taylor et al. 2021), the spread of ASF tends to spread at a faster rate in South Korea.

The spread of ASF in wild boars can be affected by the density of wild boars (Bergmann et al. 2021), mediators such as scavengers (Probst et al. 2019) and arthropods (Golnar et al. 2019), and human activities (Bergmann et al. 2021). There are no reports of scavenger or mite-mediated ASFV transmission in Korea yet. However, there have been suspected cases of human-mediated ASF spread in wild boars. A typical feature of human activity-mediated ASF spread is that ASFV-infected carcasses are found beyond local geographical boundaries (EFSA et al. 2020; EFSA et al. 2021; Podgórski and Śmietanka 2018; Taylor et al. 2021). There have been cases of likely human-mediated ASF transmission among wild boars living away from a long distance in the Czech Republic (Cukor et al. 2020), Belgium (Linden et al. 2019) and West Poland (Mazur-Panasiuk et al. 2020; Sauter-Louis et al. 2021). The newly intro- 
duced ASFV into a previously ASF-free area spreads among wild boars within the area through the carcass-mediated transmission pathway and environmental contamination (Bergmann et al. 2021; Dixon et al. 2020; Lange and Thulke 2017; Podgórski and Śmietanka 2018; Taylor et al. 2021).

In South Korea, the ASFV-infected carcass discovered in Jucheon-myeon $(37.256705,128.273151)$, Yeongwol-gun on December 28, 2020 was $89.7 \mathrm{~km}$ away from that reported on December 27, 2020 in Sancheon-myeon (37.873478, 127.616378), Hwacheon-gun, located the shortest distance from that point. The ASFV-infected carcass discovered in Gangneung-si $(37.8926,128.778)$ on February 12, 2021 was $55 \mathrm{~km}$ east of that discovered in Inje-gun (38.097705, 128.20703) on February 8, 2021. In previous studies, ASF spread by wild boar movement was estimated to be 2$5 \mathrm{~km} /$ month (Chenais et al. 2019), $1.5 \mathrm{~km} /$ month (Podgórski and Śmietanka 2018) and $<25 \mathrm{~km}$ in a year (Taylor et al. 2021). Thus, in South Korea, the medium- and long-distance spread of ASF (i.e. $>30 \mathrm{~km}$ ) is unlikely to have occurred due to dispersal of ASFV-infected wild boars; instead the other pathways such as ASF propagation by human activities are likely to be more dominant in the long-distance spread of ASF (Taylor et al. 2021).

The monthly average number of the ASFV-infected carcasses was $71.5 \pm 52.1$ cases $(n=12$, range $=24-185)$ in 2020 (January 1 to December 31), which was a little lower than $75.9 \pm 42.2$ cases $(n=10$, range $=19-168)$ in 2021 (January 1 to October 31 ), but there was no statistically significant difference between the two years $(p>0.05)$ (Fig. 2). The ASFV-infected carcasses tend to be found more in winter and spring (January to April) (Fig. 2). They appeared at the highest frequency in March (185 cases) in 2020 and February (168 cases) in 2021. In summer (August), they appeared at a rather high frequency. In EU, the proportions of ASFV-infected cases in hunted wild boars remained low throughout the year without visible seasonal patterns, but there was a clear seasonality in the proportion of ASFV-infected cases, from wild boars found dead, showing a declining trend in summer and increasing trend in winter (EFSA et al. 2021). Although there may be potential biases because the effort to search cannot be quantified only due to the quarantine policy of the country, the results show a general trend of seasonal occurrence of ASFV-infected carcasses.

\section{Habitat suitability map of ASF-infected wild boars}

We obtained area under the curve (AUC) values of 0.774 for model reliability of HSM for the ASFV-infected carcasses and 0.847 for that of hunted wild boars, indicating that the model performed better than random, thus validating the accuracy of the model (Swets 1988). The results of Maxent Jackknife test indicated that important parameters includes distance from residences, elevation and distance from roads in HSM for ASFV-infected carcasses, and elevation, distance from farmlands and distance from water system in that for the hunted wild boars (Fig. S1).

In the HSM (Fig. 3A), the area with very high probability (more than 0.75 habitat suitability index [HSI]) of finding ASFV-infected carcasses was $2.52 \%\left(285.57 \mathrm{~km}^{2}\right)$ of the analyzed area of $11,337 \mathrm{~km}^{2}$ in Gangwon-do, the area with a moderate to high probability $(0.50-0.75 \mathrm{HSI})$ was $19.06 \%$ $\left(2,161.03 \mathrm{~km}^{2}\right)$, the area with low probability $(0.25-0.5 \mathrm{HSI})$ was $48.44 \%\left(5,491.22 \mathrm{~km}^{2}\right)$, and the area with very low probability was $29.98 \%\left(3,399.07 \mathrm{~km}^{2}\right)$, respectively.

The model predicts that highly detectable areas of ASFV-infected carcasses were sporadically dispersed in western and southwestern parts of Gangwon-do, and ranged from north to south of the province along the Baekdudaegan Mountains, whereas poorly detectable areas ranged from the north to the south in the middle parts of the province (Fig. 3A).

In the HSM for the hunted wild boars (Fig. 3B), the highly suitable habitats for the hunted wild boars were found in Pyeonchang-gun, eastern part of Hoengseon-gun, eastern part of Hongchong-gun, and the western part of the Baek-

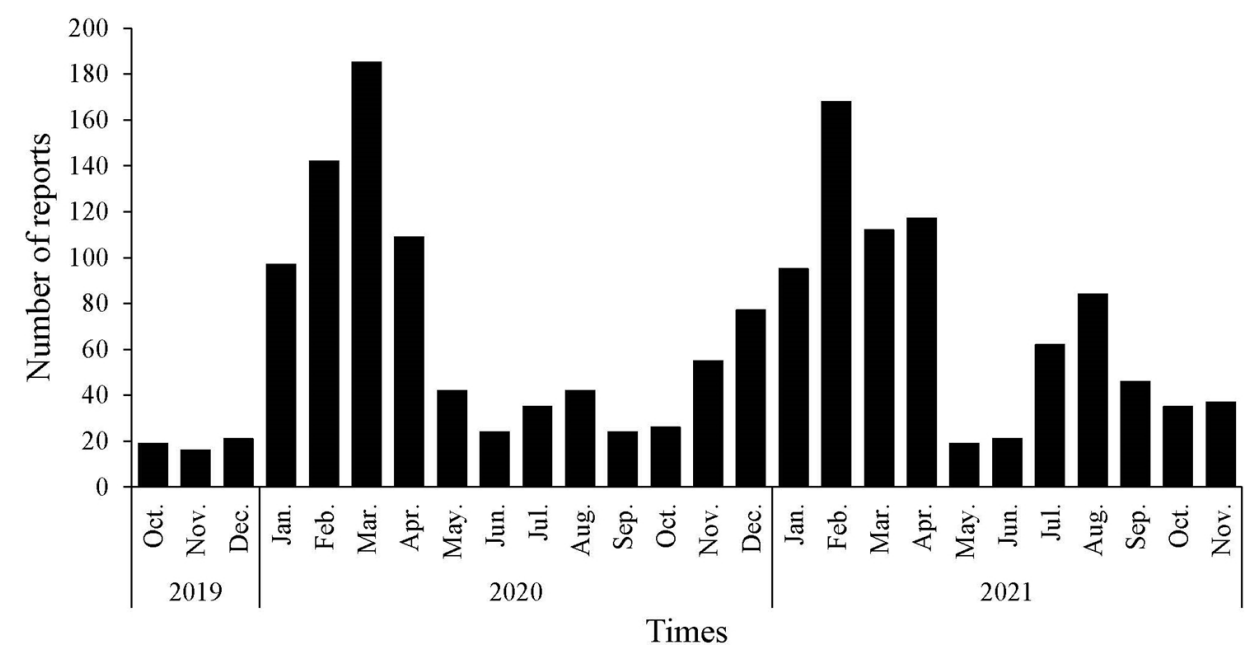

Fig. 2 The monthly discovery trend of 1,710 ASFV-infected wild boar carcasses discovered during the period from October 2, 2019 to November 20, 2021. ASFV: African swine fever virus. 

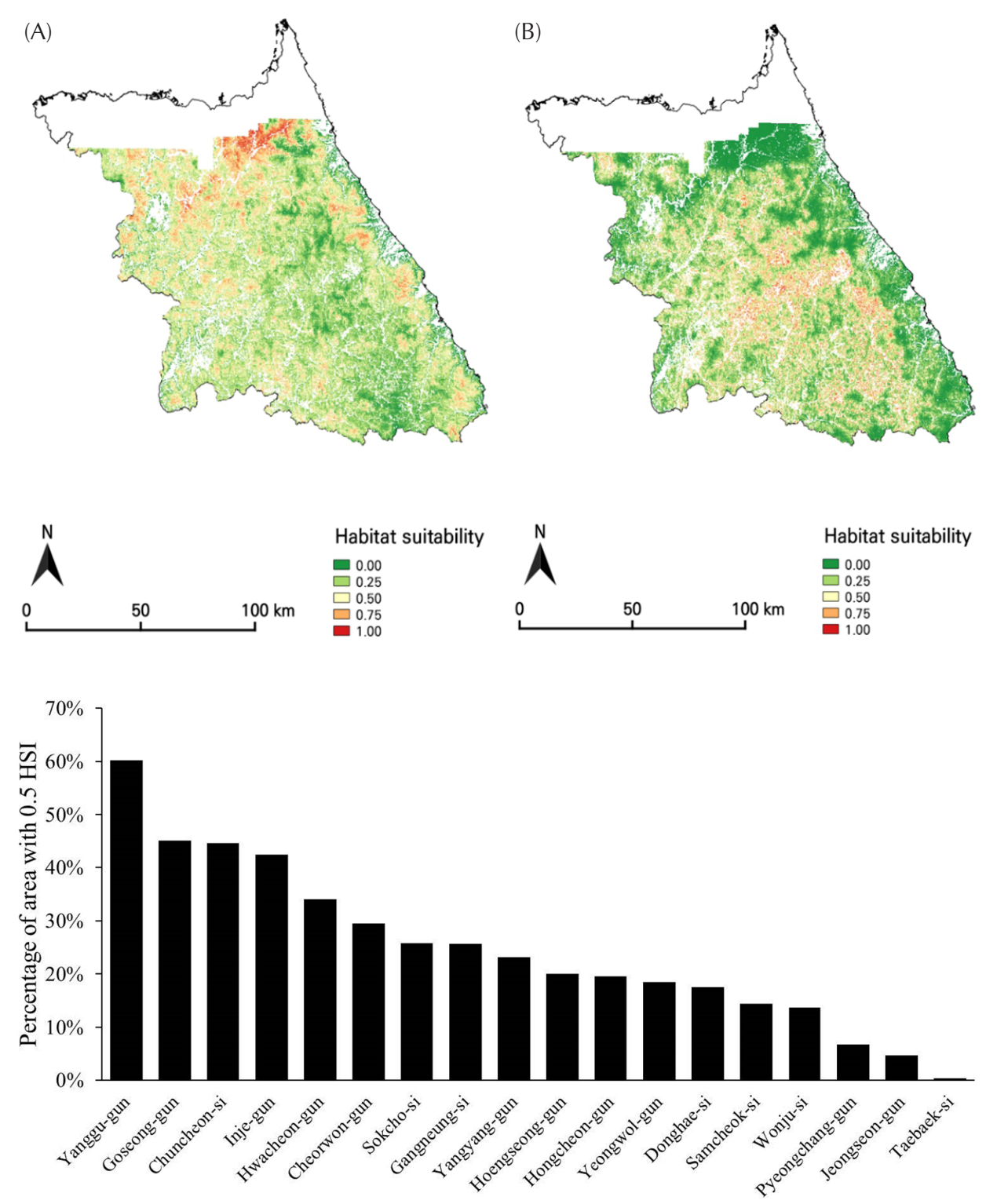

Fig. 3 Habitat suitability maps derived using the GPS coordinates of the sites where ASFV-infected wild boar carcasses (A) and the hunted wild boars (B) were found in Gangwon-do. On the map, the empty space under the northern boundary of Gangwon-do is at the places where the four forest variables of environmental variables were not provided by Korea Forest Service. GPS: global positioning system; ASFV: African swine fever virus.
Fig. 4 Suitable habitats with HSI of 0.5 or more in the habitat suitability map derived using the location GPS coordinates of ASFV-infected carcasses in each city or county in Gangwon-do (refer to Table S1). HSI: habitat suitability index; GPS: global positioning system; ASFV: African swine fever virus. dudaegan Mountains over the Pyeongchang-gun, Jeongseongun, Samcheok-si and Taebaek-si (Fig. 3B). Of the analyzed area of $11,337 \mathrm{~km}^{2}, 3.13 \%\left(354.76 \mathrm{~km}^{2}\right)$ was likely to be very highly suitable habitats for the hunted wild boars (more than 0.75 HSI ), $14.93 \%\left(1,692.32 \mathrm{~km}^{2}\right)$ was likely to be moderately to highly suitable habitats (0.50-0.75 HSI), $31.21 \%\left(3,537.82 \mathrm{~km}^{2}\right)$ was likely to be low suitable habitats (0.25-0.5 HSI), and $50.74 \%\left(5,751.98 \mathrm{~km}^{2}\right)$ was likely to be very low suitable habitats $(0.25-0.5 \mathrm{HSI})$.

\section{Implications for management}

Understanding the suitable habitats of wild boars is important in making management strategies and policy decisions, such as selection of search area for ASFV-infected carcasses, fence installation areas and hunting areas to prevent in advance the spread of ASFV-infected wild boars (Bosch et al. 2014; ENETwild Consortium et al. 2018; Lim et al. 2021; Sofaer et al. 2019).

The HSMs using raster cells with more than 5.0 HSI, based on the GPS coordinates of ASFV-infected carcasses, provides information on areas where there is a high probability of finding ASFV-infected carcasses. The suitable habitats with more than 0.5 HSI was much larger in Yanggu-gun $(60.2 \%)$ than in other cities or counties in Gangwon-do and accounted for more than $20 \%$ in 10 cities or counties (Fig. 4, Table S1).

The map shows that ASFV-infected carcasses can highly emerge in northern Gangwon-do, where ASFV-infected carcasses have frequently been found till date. If ASFV-infected wild boars spread, the infected carcasses are likely to be found from the north to the south along the Baekdudaegan Mountains and its adjacent cities and counties such as Sokcho-si, Yangyang-gun, Gangneung-gun, and Donghae-si (Fig. 5A). This spread route of ASFV-infected wild boars is well matched with highly suitable habitats of the wild boars from the HSM derived using GPS coordinates of the wild boars' trace points in the previous study (Fig. 2 in Rho 2015). 

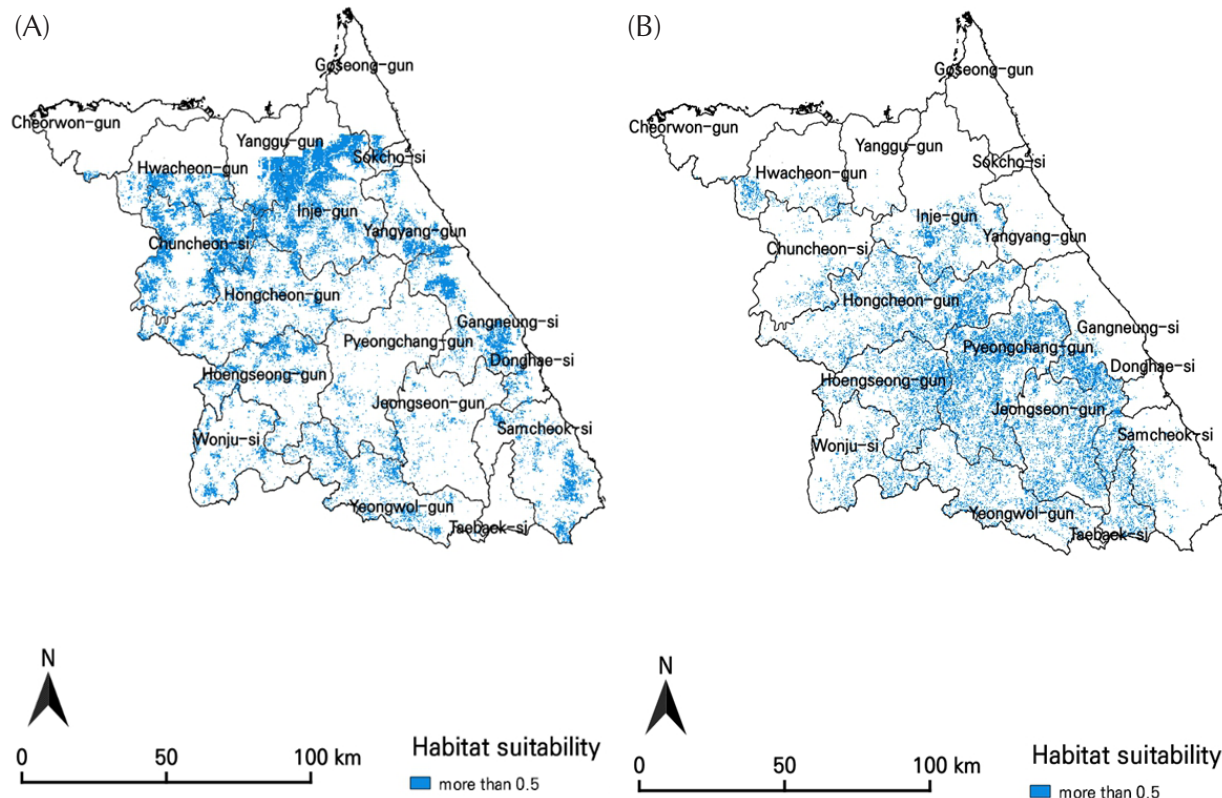

50 $\square$ more than 0.5
Habitat suitability
Fig. 5 The highly and moderately suitable habitats with HSI of more than 0.5 in the habitat suitability maps derived using the sites where ASFV-infected carcasses were found (A) and the sites where the hunted wild boars were found (B). HSI: habitat suitability index; ASFV: African swine fever virus
In the Yeongseo area, west of Gangwon-do, the ASFV-infected carcasses were more likely to be found in the northern and eastern outskirts of Chuncheon-si, the boundaries between Chuncheon-si, Hongcheon-gun, and Inje-gun, the boundaries between Pyeongchang-gun, Jeongseon-gun, and Yeongwol-gun, and the southern part of Yeongwol-gun (Fig. 5A).

According to the HSM derived using the GPS coordinates of the hunted wild boars (Fig. 5B), more moderate suitable habitats $(\geq 0.5)$ are distributed widely in the middle part of the province, revealing high suitability in most of Pyeongchang, eastern part of Hongcheong, eastern part of Heongseong, and southwestern of Gangneung. The suitable hunting sites in the HSM did not correspond to the areas where the high spread of the ASFV-infected wild boars was expected (Fig. 5).

In this study, our suitability model, based on GPS coordinates of ASFV-infected carcasses, identifies the potential habitats where ASFV-infected wild boar carcasses are likely to be found and may be an important route for ASFV to spread. The areas with high suitability predicted in this study should be given priority as survey areas to find ASFV-infected carcasses and hunting areas to reduce wild boar population.

\section{Conclusions}

This study describes the propagation trend of ASFV-infected wild boars, provides habitat suitability maps for ASFV-infected wild boars, and suggests the areas with a high probability of finding ASFV-infected carcasses. Despite active quarantine policies in Korea to prevent the spread of ASFV through wild boars, there was no significant difference in the monthly average number of ASFV-infected car- casses between 2020 and 2021. The ASFV-infected carcasses tended to be found more in winter and spring (January to April). The maximum width of ASFV-infected carcass distribution area was $222.7 \mathrm{~km}$ for about 26 months up to November 20, 2021 since the first ASF outbreak in wild boars on October 2, 2019. At least two cases (Yeongwolgun; 37.256705, 128.273151 and Gangneung-si; 37.8926, 128.778) are likely to be related to presumably any type of fomite-related translocation of the ASFV beyond the biological capacity of wild boar movement velocity. The habitat suitability model, based on GPS coordinates of ASFV-infected carcasses, identifies potential habitats where ASFV-infected carcasses are likely to be found and may be an important route for ASFV to spread. Among the ASFfree areas, the areas with high suitability predicted in this study should be given priority as survey areas to find ASFinfected carcasses and hunting areas to reduce wild boar populations.

\section{Supplementary Information}

Supplementary information accompanies this paper at https://doi.org/10.5141/jee.22.006.

Table S1. The ratio of area with HSI of 0.5 or more in the habitat suitability map using the location coordinates of ASF-infected carcasses in each city or county in Gangwon-do. Fig. S1. Maxent Jackknife test of the environmental variables for habitat suitability map of ASFV-infected carcasses and the hunted wild boars.

\section{Abbreviations}

ASF: African swine fever

ASFV: African swine fever virus

EU: European Union 
GPS: Global positioning system

HIS: Habitat suitability index

HSM: Habitat suitability map

MAFRA: Ministry of Agriculture, Food and Rural Affairs

\section{Acknowledgements}

We are grateful to Joong Yeol Park, So Hyeon Han, Ju Hui Choi, Kyeong Tae Choi, Ji Hyeon Kweon and Jin Geum Kim for helping data collection. We also thank anonymous reviewers for helpful comments on the drafts of this article.

\section{Authors' contributions}

SJL collected and analyzed data, wrote original draft. HN collected and analyzed data. NHK collected data. YO acquired the research grant, and conceptualized and supervised the research. YCP conceptualized and supervised the research, and reviewed and edited the draft. The authors read and approved the final manuscript.

\section{Funding}

This work was supported by Korea Institute of Planning and Evaluation for Technology in Food, Agriculture and Forestry (IPET) through Animal Disease Management Technology Development Program, funded by Ministry of Agriculture, Food and Rural Affairs (MAFRA) (320067-02-1-CG000) and Kangwon National University 2019.

\section{Availability of data and materials}

The datasets generated during and/or analyzed during the current study are available from the corresponding author on reasonable request.

\section{Ethics approval and consent to participate \\ Not applicable.}

\section{Consent for publication \\ Not applicable.}

\section{Competing interests}

The authors declare that they have no competing interests.

\section{References}

Bergmann H, Schulz K, Conraths FJ, Sauter-Louis C. A review of environmental risk factors for African swine fever in European wild boar. Animals (Basel). 2021;11(9):2692. https://doi.org/10.3390/ani11092692.

Bosch J, Torre A, Alexandrov T, Iglesias I, Miteva A, Muñoz M. Can habitat suitability predict the presence of wild boar? Suitable land uses vs. georeferenced data in Bulgaria. Folia Zool. 2014;63(3):194205. https://doi.org/10.25225/fozo.v63.i3.a7.2014.

Chenais E, Depner K, Guberti V, Dietze K, Viltrop A, Ståhl K. Epidemiological considerations on African swine fever in Europe 2014-2018. Porcine Health Manag. 2019;5:6. https://doi.org/10.1186/s40813018-0109-2.
Cukor J, Linda R, Václavek P, Šatrán P, Mahlerová K, Vacek Z, et al. Wild boar deathbed choice in relation to ASF: are there any differences between positive and negative carcasses? Prev Vet Med. 2020;177:104943. https://doi.org/10.1016/j.prevetmed.2020.104943.

Dixon LK, Chapman DA, Netherton CL, Upton C. African swine fever virus replication and genomics. Virus Res. 2013;173(1):3-14. https:// doi.org/10.1016/j.virusres.2012.10.020.

Dixon LK, Stahl K, Jori F, Vial L, Pfeiffer DU. African swine fever epidemiology and control. Annu Rev Anim Biosci. 2020;8:221-46. https://doi.org/10.1146/annurev-animal-021419-083741.

EFSA (European Food Safety Authority), Anette B, Anette B, Theodora CV, Klaus D, Daniel D, et al. Epidemiological analyses of African swine fever in the European Union (November 2018 to October 2019). EFSA J. 2020;18(1):e05996. https://doi.org/10.2903/j.efsa. 2021.5996

ENETwild Consortium, Croft S, Smith G, Acevedo P, Vicente J. Wild boar in focus: review of existing models on spatial distribution and density of wild boar and proposal for next steps. EFSA Support Publ. 2018;15(10):1490E. https://doi.org/10.2903/sp.efsa.2018.EN-1490.

European Food Safety Authority (EFSA), Desmecht D, Gerbier G, Gortázar Schmidt C, Grigaliuniene V, Helyes G, et al. Epidemiological analysis of African swine fever in the European Union (September 2019 to August 2020). EFSA J. 2021;19(5):e06572. https://doi. org/10.2903/j.efsa.2021.6572.

Fischer M, Hühr J, Blome S, Conraths FJ, Probst C. Stability of African swine fever virus in carcasses of domestic pigs and wild boar experimentally infected with the ASFV "Estonia 2014" isolate. Viruses. 2020;12(10):1118. https://doi.org/10.3390/v12101118.

Golnar AJ, Martin E, Wormington JD, Kading RC, Teel PD, Hamer SA, et al. Reviewing the potential vectors and hosts of African swine fever virus transmission in the United States. Vector Borne Zoonotic Dis. 2019;19(7):512-24. https://doi.org/10.1089/vbz.2018.2387.

Korea Forest Service. 2020 Forest Basic Statistics. 2021. https://kfss.forest.go.kr/stat/ptl/main/main.do. Accessed 10 Dec 2021.

Lange M, Thulke HH. Elucidating transmission parameters of African swine fever through wild boar carcasses by combining spatio-temporal notification data and agent-based modelling. Stoch Environ Res Risk Assess. 2017;31:379-91. https://doi.org/10.1007/s00477-016$1358-8$.

Lim JS, Vergne T, Pak SI, Kim E. Modelling the spatial distribution of ASF-positive wild boar carcasses in South Korea using 2019-2020 national surveillance data. Animals (Basel). 2021;11(5):1208. https:// doi.org/10.3390/ani11051208.

Lim SJ, Kim JY, Park YC. Analysis of habitat characteristics of leopard cat (Prionailurus bengalensis) in Odaesan National Park. J Agric Life Sci. 2015;49(3):99-111. https://doi.org/10.14397/jals.2015.49.3.99.

Linden A, Licoppe A, Volpe R, Paternostre J, Lesenfants C, Cassart D, et al. Summer 2018: African swine fever virus hits north-western Europe. Transbound Emerg Dis. 2019;66(1):54-5. https://doi.org/10. 1111/tbed.13047.

Mazur-Panasiuk N, Walczak M, Juszkiewicz M, Woźniakowski G. The spillover of African swine fever in Western Poland revealed its estimated origin on the basis of O174L, K145R, MGF 505-5R and IGR I73R/I329L genomic sequences. Viruses. 2020;12(10):1094. https:// 
doi.org/10.3390/v12101094.

Mazur-Panasiuk N, Żmudzki J, Woźniakowski G. African swine fever virus - persistence in different environmental conditions and the possibility of its indirect transmission. J Vet Res. 2019;63(3):303-10. https://doi.org/10.2478/jvetres-2019-0058.

Mur L, Martínez-López B, Sánchez-Vizcaíno JM. Risk of African swine fever introduction into the European Union through transport-associated routes: returning trucks and waste from international ships and planes. BMC Vet Res. 2012;8:149. https://doi.org/10.1186/17466148-8-149.

OIE. African Swine Fever (ASF) Report N47: 2016-2020. 2021. https:// www.oie.int/app/uploads/2021/03/report-47-global-situation-asf.pdf. Accessed 22 Dec 2021.

Olesen AS, Lohse L, Boklund A, Halasa T, Gallardo C, Pejsak Z, et al. Transmission of African swine fever virus from infected pigs by direct contact and aerosol routes. Vet Microbiol. 2017;211:92-102. https://doi.org/10.1016/j.vetmic.2017.10.004.

Plowright W, Parker J, Peirce MA. African swine fever virus in ticks (Ornithodoros moubata, Murray) collected from animal burrows in Tanzania. Nature. 1969;221(5185):1071-3. https://doi.org/10.1038/2211071a0.

Podgórski T, Śmietanka K. Do wild boar movements drive the spread of African swine fever? Transbound Emerg Dis. 2018;65(6):1588-96. https://doi.org/10.1111/tbed.12910.

Probst C, Gethmann J, Amler S, Globig A, Knoll B, Conraths FJ. The potential role of scavengers in spreading African swine fever among wild boar. Sci Rep. 2019;9(1):11450. https://doi.org/10.1038/s41598019-47623-5. Erratum in: Sci Rep. 2020;10(1):8624. https://doi. org/10.1038/s41598-020-65547-3.

Rho P. Using habitat suitability model for the wild boar (Sus scrofa Linnaeus) to select wildlife passage sites in extensively disturbed tem- perate forests. J Ecol Environ. 2015;38(2):163-73. https://doi.org/10. 5141/ecoenv.2015.018.

Sauter-Louis C, Forth JH, Probst C, Staubach C, Hlinak A, Rudovsky A, et al. Joining the club: first detection of African swine fever in wild boar in Germany. Transbound Emerg Dis. 2021;68(4):1744-52. https://doi.org/10.1111/tbed.13890.

Seo CW, Choi TY, Choi YS, Kim DY. A study on wildlife habitat suitability modeling for Goral (Nemorhaedus caudatus raddeanus) in Seoraksan National Park. J Korean Environ Res Reveg Tech. 2008;11(3):28-38. (in Korean with English abstract).

Sofaer H, Jarnevich C, Pearse I, Smyth R, Auer S, Cook G, et al. Development and delivery of species distribution models to inform decision-making. BioScience. 2019;69(7):544-57. https://doi.org/10. 1093/biosci/biz045.

Song WK, Kim EY. A comparison of machine learning species distribution methods for habitat analysis of the Korea water deer (Hydropotes inermis argyropus). Korean J Remote Sens. 2012;28(1):17180. https://doi.org/10.7780/kjrs.2012.28.1.171.

Swets JA. Measuring the accuracy of diagnostic systems. Science. 1988;240(4857):1285-93. https://doi.org/10.1126/science.3287615.

Taylor RA, Podgórski T, Simons RRL, Ip S, Gale P, Kelly LA, et al. Predicting spread and effective control measures for African swine fever-should we blame the boars? Transbound Emerg Dis. 2021;68(2): 397-416. https://doi.org/10.1111/tbed.13690.

Zakharova OI, Titov IA, Gogin AE, Sevskikh TA, Korennoy FI, Kolbasov DV, et al. African swine fever in the Russian Far East (20192020): spatio-temporal analysis and implications for wild ungulates. Front Vet Sci. 2021;8:723081. https://doi.org/10.3389/fvets.2021. 723081. 